\title{
Interventions Delivered in Clinical Settings are Effective in Reducing Risk of HIV Transmission Among People Living with HIV: Results from the Health Resources and Services Administration (HRSA)'s Special Projects of National Significance Initiative
}

\author{
Janet J. Myers $\cdot$ Starley B. Shade $\cdot$ Carol Dawson Rose $\cdot$ \\ Kimberly Koester • Andre Maiorana - Faye E. Malitz • \\ Jennifer Bie - Mi-Suk Kang-Dufour • Stephen F. Morin \\ Published online: 13 March 2010 \\ (C) The Author(s) 2010. This article is published with open access at Springerlink.com
}

\begin{abstract}
To support expanded prevention services for people living with HIV, the US Health Resources and Services Administration (HRSA) sponsored a 5-year initiative to test whether interventions delivered in clinical settings were effective in reducing HIV transmission risk among HIV-infected patients. Across 13 demonstration sites, patients were randomized to one of four conditions. All interventions were associated with reduced unprotected vaginal and/or anal intercourse with persons of HIVuninfected or unknown status among the 3,556 participating patients. Compared to the standard of care, patients assigned to receive interventions from medical care providers reported a significant decrease in risk after 12 months of participation. Patients receiving prevention services from health educators, social workers or paraprofessional HIV-infected peers reported significant reduction in risk at 6 months, but not at 12 months. While clinics have a choice of effective models for implementing prevention programs for their HIV-infected patients, medical provider-delivered methods are comparatively robust.
\end{abstract}

Keywords HIV Prevention with positives . Clinic-based HIV prevention - HIV risk reduction . Interventions $\cdot$ Study outcomes

J. J. Myers $(\bowtie)$ - S. B. Shade · C. D. Rose · K. Koester A. Maiorana - J. Bie - M.-S. Kang-Dufour - S. F. Morin Center for AIDS Prevention Studies, University of California, San Francisco, 50 Beale Street, Suite 1300, San Francisco, CA 94105, USA

e-mail: janet.myers@ucsf.edu

F. E. Malitz

Health Resources and Services Administration, HIV/AIDS

Bureau, 5600 Fishers Lane, Rockville, MD 20857, USA

\section{Introduction}

HIV primary care clinics are an important setting in which to deliver behavioral HIV prevention interventions because HIV-infected individuals are likely to be seen in these settings for regular care four times per year or more. A growing literature on the importance of delivering prevention interventions to HIV-infected patients (prevention with positives or PWP) demonstrates that medical care providers can help patients reduce risk [1-3]. Other types of prevention service providers have also delivered effective programs for HIV-infected individuals. For example, prevention counselors [4], group facilitators [5] and HIVinfected peers [6] have helped HIV-infected individuals reduce their risk. However, no study has compared the effectiveness of HIV prevention interventions delivered in clinical settings by different types of providers to determine which interventions work best in helping HIV-infected patients reduce their risk of transmitting HIV to others.

To determine the relative effectiveness of different types of prevention programs delivered in clinical settings, the Special Projects of National Significance (SPNS) program of the Health Resources and Services Administration (HRSA) funded 15 demonstration sites and an evaluation and support center to implement and evaluate prevention interventions for HIV-infected people who were seen in clinical settings [7]. Sites were required to use intervention approaches that were based on models that had been shown to be effective in prior research. Most sites chose interventions based on the well-tested health behavior theories of the Transtheoretical Model [8], motivational interviewing [9] and/or harm reduction [10]. Table 1 provides an overview of the theoretical models used by participating sites. All sites used formative research and expert 


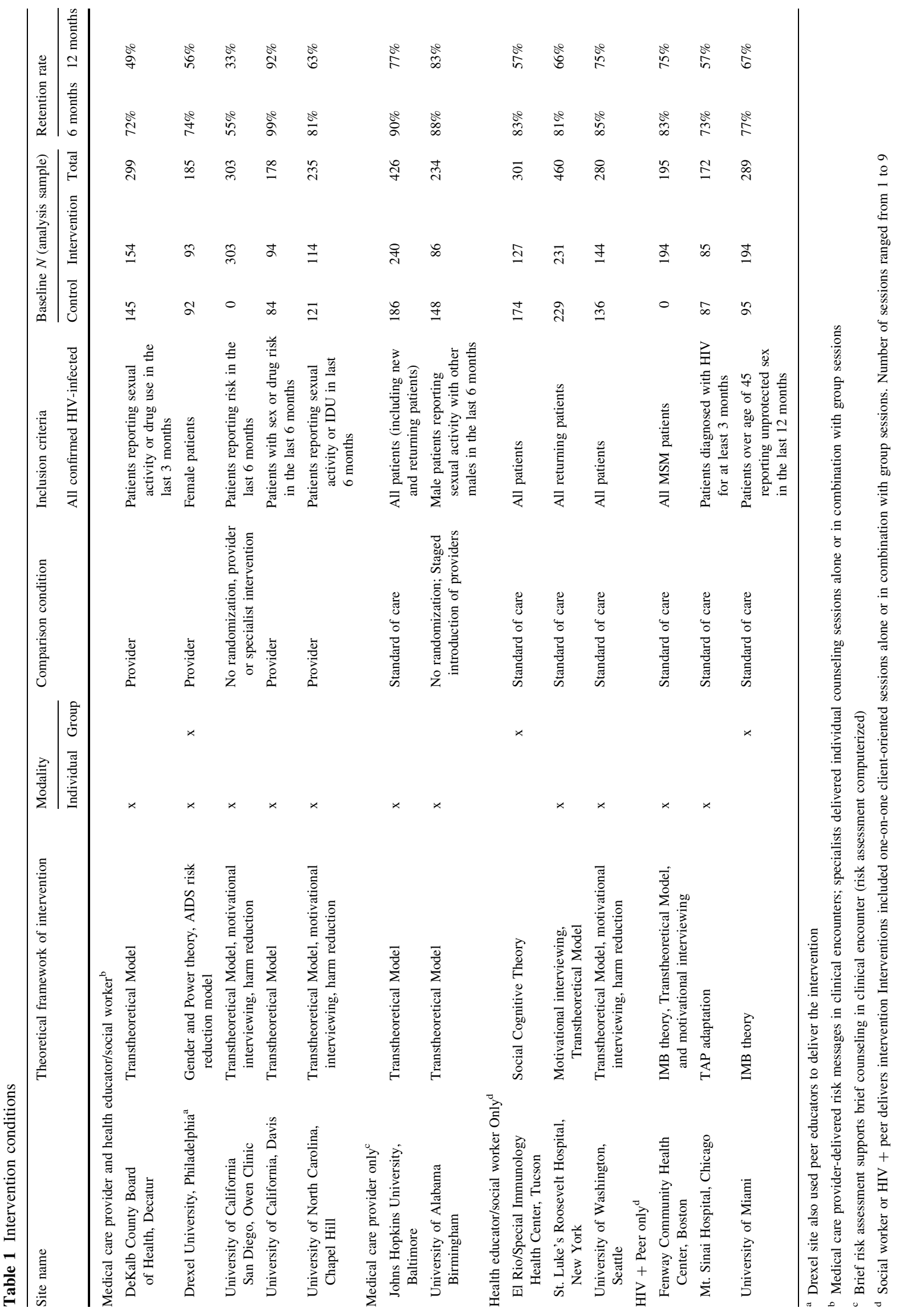


consultation to tailor these approaches to the local client population and clinical setting. Detail on the tailoring processes is available elsewhere [11].

Sites also used a variety of provider types to deliver intervention services (Table 1). Specifically, in this study, medical care provider-delivered interventions used brief risk assessment via computer to support clinicians in delivering brief counseling during routine medical visits. In some sites, social workers or health educators delivered HIV-prevention counseling. Health educators and social workers worked alone, with medical providers or with HIVinfected peers to deliver interventions. Most often, interventions involved one-on-one client-oriented sessions. In some sites, these were combined with group sessions. The number of sessions defining each intervention ranged from one to nine. In sites where prevention messages were delivered by more than one type of provider ("multi-provider interventions"), medical care providers delivered prevention messages in clinical encounters; health educators, social workers or peer educators also conducted risk assessments and delivered individual counseling sessions alone or in combination with group sessions. Table 1 also provides a description of the provider characteristics in each participating site.

This paper describes the outcomes of the project across all sites. We present findings describing whether and to what degree behavioral interventions in primary care settings help HIV-infected patients reduce the risk of transmitting HIV. We also describe the relative HIV risk reduction among patients receiving medical care providerled interventions, interventions delivered by health educators, social workers or paraprofessional HIV-infected peers serving as "HIV prevention specialists" or interventions delivered by a medical provider and an HIV prevention specialist (multi-provider interventions) compared to patients receiving the standard of care.

\section{Methods}

Interventions and survey interviews were conducted in funded sites between April 2004 and December 2006. Sites were selected based on a competitive application process and were located in Chapel Hill, NC; Boston, MA; Baltimore, MD; New York City, NY; Seattle, WA; Sacramento, CA; San Diego, CA; Birmingham, AL; Philadelphia, PA; Decatur, GA; Miami, FL; Chicago, IL; and Tucson, AZ. Some sites had more than one clinic, and all participating clinics received funding from the federal Ryan White Program with 300 patients or more. Each site required local human research subjects approval in addition to the approval obtained from the University of California, San Francisco, which served as the cross-site evaluation center.
Although 15 sites were funded through this initiative, the analysis presented here is restricted to data from 13 of the sites. The Office of AIDS Policy and Planning, Los Angeles site was omitted because study investigators used different interviewing procedures and the resulting data were systematically different from the other 14 sites. Data from the Whitman Walker Clinic site in Washington, D.C. was omitted because there was an interruption in the study due to fiscal hardship within the organization; this interruption resulted in information not being available on patient receipt of the intervention and assignment to the intervention or assessment-only conditions.

\section{Study Population}

A total of 3,556 HIV-infected patients completed audiocomputer assisted self interviews (ACASI) at baseline. These interviews included standardized cross-site questions and additional questions that a site's study team added and used in local analysis. The ACASI survey was programmed using Questionnaire Development System version 2.0 by Nova Research Company (Bethesda, MD). Inclusion criteria were HIV-infected status, receipt of primary care at the clinic, age of 18 years or older, and ability to provide informed consent. For the cross-site evaluation, we translated the interview instrument into Spanish; however, this version was used at only one site. All other respondents were English-speaking. Sites had the option of adding additional inclusion requirements, which are summarized in Table 1. The cross-site evaluation survey took approximately 30 minutes to complete.

Recruitment and screening of potential respondents was undertaken exclusively in medical clinics serving HIVinfected clients. Sites used a variety of recruitment materials including brochures, posters, and project descriptions, as well as direct contact by study staff in clinics. Interested patients were briefly screened by project personnel to determine their self-reported HIV status as well as basic demographic and contact information. Then, eligible participants were scheduled for a baseline interview. Screening took place in a private setting, usually in a room or quiet place in the clinic. Most sites used incentives worth approximately $\$ 25$ such as cash, a grocery voucher, or a gift certificate, to encourage participation in the evaluation portion of the project. Participants were not given incentives to attend intervention visits.

\section{Randomization}

Patients were individually randomized to an intervention group or a comparison condition on a site-by-site basis. Patients in the intervention group were assigned to receive 
interventions from their medical care providers during routine HIV care visits alone or in combination with services delivered by health educators, case managers or social workers or by HIV-infected peers trained to conduct HIV prevention counseling. Providers delivered interventions as stand alone sessions or in conjunction with routine clinical visits. Social workers, health educators and HIVinfected peers hired by these projects served the same role. In sites that included either medical provider-delivered or prevention specialist-delivered interventions, patients in the comparison group received the standard of care, routine HIV care visits by their medical care provider. Some sites' interventions used a combination of approaches so that patients received prevention counseling from both medical providers and prevention specialists. In these multi-provider sites, patients in the comparison group received medical provider-delivered prevention counseling only. For this analysis, data were combined from patients in sites where the comparison group received the standard of care with those in multi-provider sites where the intervention condition was medical provider prevention only. Intervention and comparison types are summarized in Table 1.

\section{Study Measures}

\section{Demographic Characteristics and Health Status Indicators}

Detailed background and demographic items included participant's age, race/ethnicity, gender, self-identified sexual orientation, relationship status, educational level, employment status, and income. In addition, self-reported health status indicators were assessed including: most recent CD4 count, HIV viral load, and current use of antiretroviral medication.

\section{Sexual Behavior}

Participants were asked to report sexual behavior over a 6 month recall period. Separate but equivalent versions of questions were developed for men and women, each with language tailored to be consistent with the participant's gender and sexual orientation. Participants were asked to provide the number of times they had engaged in insertive or receptive vaginal and/or anal sex with HIV-infected partners, HIV-uninfected partners and partners of unknown HIV status. Participants were also asked about the number of times they had used condoms (male or female) from the beginning to the end of penetration and the number of times sex was unprotected. Unprotected sex was limited in the questioning to any act of insertive or receptive anal or vaginal intercourse in which a participant did not use a condom, a definition that excludes risk acts produced by accidental condom slippage or breakage.

\section{Sexual Transmission Risk}

Transmission risk acts were defined as reports of unprotected anal or vaginal intercourse without the use of a condom with any HIV-uninfected or unknown status partners. These acts were dichotomized into, "Yes" or "No." Our outcome indicator does not include sex with HIV-infected partners because superinfection has not been observed in individuals such as those participating in this project, primarily characterized as having been infected for more than 3 years, engaged in care and on antiretroviral drugs.

\section{Substance Use}

Use of legal and illegal substances was assessed over a 3 month recall period. Items included alcohol, cocaine/ crack, sedatives, tranquilizers, stimulants (such as crystal methamphetamine), analgesics, inhalants, marijuana, hallucinogens, and heroin. Use of injected drugs was assessed over the past 30 days. Items included frequency of injection and whether a participant had lent a needle to someone else after using it.

Patients repeated the assessments at 6-month intervals for 12 months after their initial baseline assessment and randomization.

\section{Statistical Analysis}

We compare participant characteristics, sexual behavior and substance use at study entry by intervention type using chi-square tests of homogeneity. Patients assigned to peer or health educator/social worker-led interventions responded similarly to the interventions with respect to their sexual transmission risk behavior and were combined in the analysis into a category entitled "prevention specialists." We present the prevalence of sexual transmission risk behavior among participants in each intervention type over the 12-month assessment period graphically (Fig. 1). We compare the odds of sexual transmission risk behavior among participants in each intervention type to the odds of sexual transmission risk behavior among participants in the standard of care group at each time point using generalized estimating equations (GEE). In this model, we estimate the odds of sexual transmission risk behavior among participants in each intervention type at study entry, 6 months and 12 months using indicator variables for each intervention type at each time point. In order to account for clustering of participant behavior by intervention site, the GEE models adjust for sexual transmission risk behavior at study entry, as well as the correlation among participants at each site and within intervention groups. 


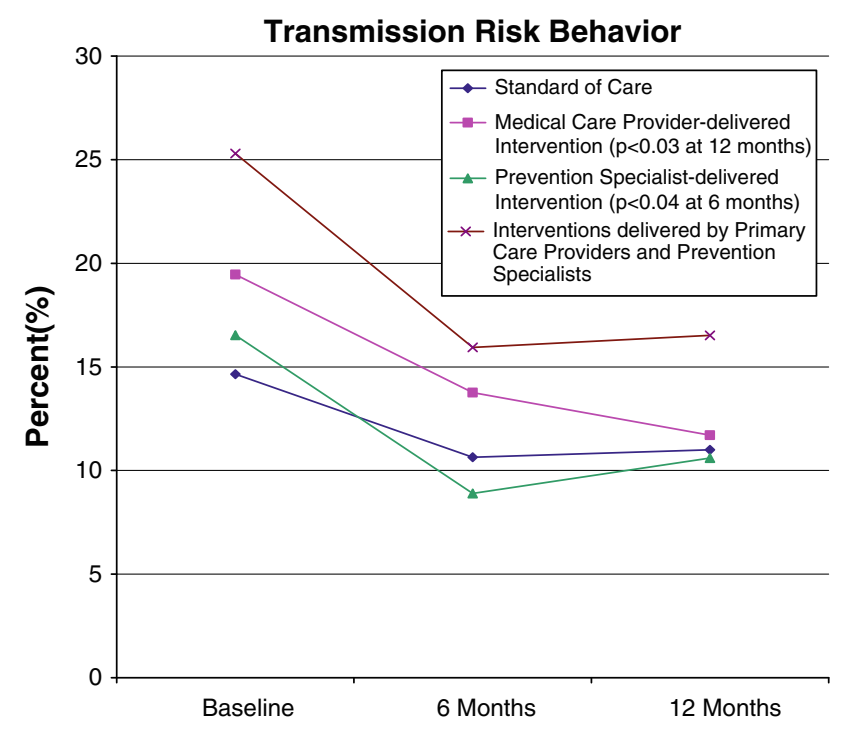

Fig. 1 Transmission Risk Behavior across 3 time points for Medical Care Provider-delivered, Prevention specialist-delivered, Multi-Provider Intervention and Standard of Care Groups

Because randomization occurred at the level of individual sites, rather than across the study as a whole, we employed inverse probability of treatment weighting (IPTW) to account for the effect of differences in the patient populations across intervention types on the observed effect of each intervention type [12, 13]. This method has the effect of creating samples in each intervention type that have been weighted to balance out the distribution of demographic and other patient characteristics associated with sexual transmission risk behavior. This creates a weighed population where characteristics of individuals are similar across all intervention types. Estimated parameters thus reflect the effect of each intervention type that would have been seen if it were possible to randomize the treatments across settings.

To estimate these weights, we used categorical logistic regression to estimate the probability of being assigned to each intervention type, given an individual's demographic characteristics (age, gender, sexual orientation, race/ethnicity) and reported substance use (alcohol, speed and/or injecting drug use) at baseline. Factors in the model were chosen based on their association with the primary outcome at baseline, which are described in our paper on predictors of risk in this population [14]. The inverses of these probabilities were then applied as weights to the GEE model described above. However, application of these weights impacted the observed results by less than $1 \%$. Therefore, they were not retained in the final model.

Because loss to follow-up was different across study sites and treatment arms, we used additional weighting methods to assess the impact of loss to follow-up on observed study results. These methods have the effect of weighting the data at each follow-up to resemble the sample at baseline with respect to factors associated with sexual transmission risk. Estimated parameters thus reflect the expected effect of each intervention type had no lossto-follow-up occurred. To estimate these weights, we used logistic regression to estimate the probability of remaining in the study at each time point, given an individual's demographic and substance use at baseline as defined above. The inverse of these probabilities were then applied as inverse probabilities of censoring weights (IPCW) to the final GEE model described above. Application of these results did impact the observed results. Therefore these weights were retained in the final model. The combination of the GEE model and the weighting procedures allows us to generate estimates for the effect of treatment at a population level accounting for differences across sites and the correlation of individuals results within sites. All analyses were implemented in SAS (version 9.1, SAS Institute Inc, Cary, NC).

\section{Results}

\section{Participant Characteristics at Study Entry}

Participant characteristics by intervention model are presented in Table 2. Of the 3,556 participants completing baseline assessments, most participants were men (70\%) and over 40 years of age (64\%). Forty-eight percent were African American, 37\% were Caucasian, $11 \%$ were Hispanic or Latino and $4 \%$ were of mixed or other race/ethnicities. Similar proportions of participants identified as gay (44\%) and heterosexual (45\%), while fewer identified as bisexual $(8 \%)$ or of unknown sexual orientation (2\%). Approximately half of participants had a high school degree and $39 \%$ had a paying job. HIV-related health status among participants was good with $60 \%$ reporting CD4+ T cell counts greater than $200\left(\right.$ cells $\left./ \mathrm{mm}^{3}\right)$ and $15 \%$ reporting CD4+ T cell counts less than $200\left(\right.$ cells $\left./ \mathrm{mm}^{3}\right)$. One quarter of participants did not know the value of their most recent CD4+ T cell count. One-third of participants reported having an undetectable viral load, 53\% reported having a detectable viral load and $12 \%$ did not know the value of their most recent viral load test. Two-thirds of participants reported current use of antiretroviral therapy.

Sexual Behavior and Drug Use in the 6 Months Prior to Study Entry

Table 3 presents the sexual risk characteristics at baseline of 3,556 patients across intervention types. Overall, $77 \%$ of patients reported at least one sexual partner in the previous 6 months $(n=2,723)$. Among patients reporting sexual 
Table 2 Characteristics at baseline of 3,556 study participants

\begin{tabular}{|c|c|c|c|c|c|c|}
\hline & $\begin{array}{l}\text { Standard of care } \\
(N=1055)\end{array}$ & $\begin{array}{l}\text { Medical care provider } \\
\text { intervention } \\
(N=768)\end{array}$ & $\begin{array}{l}\text { Prevention specialist } \\
\text { intervention } \\
(N=975)\end{array}$ & $\begin{array}{l}\text { Multi-provider } \\
\text { Intervention } \\
(N=758)\end{array}$ & $\chi^{2}$ & $P \leq$ \\
\hline Gender & & & & & 25.1 & 0.001 \\
\hline Male & $781(74)$ & $490(64)$ & $705(72)$ & $530(72)$ & & \\
\hline Female & $269(26)$ & $270(35)$ & $265(27)$ & $224(27)$ & & \\
\hline Transgender & $5(0.5)$ & $8(1)$ & $5(1)$ & $4(1)$ & & \\
\hline Race/ethnicity & & & & & 97.5 & 0.001 \\
\hline White & $410(39)$ & $282(37)$ & $332(25)$ & $298(22)$ & & \\
\hline African American & $461(43)$ & $445(58)$ & $454(26)$ & $454(26)$ & & \\
\hline Hispanic/Latino & $142(13)$ & $22(3)$ & $140(25)$ & $125(23)$ & & \\
\hline Other & $42(4)$ & $19(2)$ & $49(31)$ & $23(15)$ & & \\
\hline Sexual orientation & & & & & 23.6 & 0.01 \\
\hline Homosexual & $487(46)$ & $313(41)$ & $405(42)$ & $373(49)$ & & \\
\hline Bisexual & $86(8)$ & $65(8)$ & $73(8)$ & $70(9)$ & & \\
\hline Heterosexual & $453(43)$ & $371(48)$ & $478(49)$ & 297 (39) & & \\
\hline Unknown/No answer & $29(3)$ & $19(2)$ & $19(2)$ & $18(2)$ & & \\
\hline Age & & & & & 79.7 & 0.001 \\
\hline 39 or less & $335(32)$ & $345(45)$ & $271(28)$ & $327(43)$ & & \\
\hline 40 or more & $720(68)$ & $423(55)$ & $704(72)$ & $431(57)$ & & \\
\hline Education completed & & & & & 5.2 & 0.16 \\
\hline High school or less & $540(51)$ & 377 (49) & $524(54)$ & $371(49)$ & & \\
\hline Some college or more & $515(49)$ & $391(51)$ & $451(46)$ & $387(51)$ & & \\
\hline Unknown/No answer & $0(0)$ & $0(0)$ & $0(0)$ & $0(0)$ & & \\
\hline Employment & & & & & 36.6 & 0.001 \\
\hline Unemployed & $644(61)$ & $413(54)$ & $648(65)$ & $478(63)$ & & \\
\hline Employed & $411(39)$ & $355(46)$ & $324(33)$ & $279(37)$ & & \\
\hline Unknown/No answer & $0(0)$ & $0(0)$ & $3(0)$ & $1(0)$ & & \\
\hline CD4 cell count & & & & & 10.1 & 0.12 \\
\hline Below 200 & $152(14)$ & $109(14)$ & $154(16)$ & $120(16)$ & & \\
\hline 200 or above & $622(59)$ & $461(60)$ & $575(59)$ & $481(64)$ & & \\
\hline Unknown/No answer & $281(27)$ & $198(26)$ & $246(25)$ & $157(21)$ & & \\
\hline Most recent viral load & & & & & 68.7 & 0.001 \\
\hline Undetectable & $381(36)$ & $216(28)$ & $418(43)$ & $219(29)$ & & \\
\hline Detectable & $537(51)$ & $445(58)$ & $469(48)$ & $448(59)$ & & \\
\hline Unknown/no answer & $137(13)$ & 107 (14) & $88(9)$ & $91(13)$ & & \\
\hline Currently on ART & & & & & 29.1 & 0.001 \\
\hline No & $151(14)$ & $126(16)$ & $108(11)$ & $129(17)$ & & \\
\hline Yes & $711(68)$ & $481(63)$ & $694(71)$ & $458(60)$ & & \\
\hline Unknown/no answer & $193(18)$ & $161(21)$ & $173(18)$ & $171(23)$ & & \\
\hline
\end{tabular}

activity at baseline, the mean number of partners overall was five (standard deviation $=11$ ) and the median was 2 $(25-75 \%=1-3)$. Thirty-nine percent of sexually active patients reported two or more partners at baseline. Of these, $1,421(52 \%)$ reported one or more HIV-infected partners, $1,324(49 \%)$ reported one or more HIV-uninfected partners and $847(31 \%)$ reported one or more partners of unknown serostatus. Thirty percent reported any vaginal sex.
Twenty-seven percent reported anal insertive sex and 32\% anal receptive sex. Twenty-four percent of participants reported anal insertive sex with male sexual partners, and $3 \%$ reported anal insertive sex with female sexual partners. Eleven percent of patients reported unprotected vaginal or anal sex with an uninfected partner. Twenty-one percent reported unprotected vaginal or anal sex with an HIVinfected partner and $10 \%$ reported unprotected vaginal or 
Table 3 Risk behaviors at baseline of 3,556 study participants

\begin{tabular}{|c|c|c|c|c|c|c|}
\hline & $\begin{array}{l}\text { Standard of care } \\
(N=1055)\end{array}$ & $\begin{array}{l}\text { Medical care } \\
\text { provider intervention } \\
(N=768)\end{array}$ & $\begin{array}{l}\text { Prevention specialist } \\
\text { intervention } \\
(N=975)\end{array}$ & $\begin{array}{l}\text { Multi-provider } \\
\text { intervention } \\
(N=758)\end{array}$ & $\chi^{2}$ & $P$ \\
\hline \multicolumn{7}{|l|}{ Sexual activity in last 6 months } \\
\hline Sexually active & $771(73)$ & $621(81)$ & $718(74)$ & $613(82)$ & 29.1 & 0.001 \\
\hline \multicolumn{7}{|l|}{ Sexual partners ${ }^{\mathrm{a}}$} \\
\hline Mean (SD) number of partners & $3.8(9.3)$ & $3.6(7.8)$ & $4.8(12.4)$ & $6.2(12.5)$ & & \\
\hline Median [IQR] & $1(1-3)$ & $1(1-3)$ & $2(1-4)$ & $2(1-5)$ & & \\
\hline Two or more sex partners & $379(49)$ & $298(48)$ & $363(51)$ & $338(55)$ & 7.5 & 0.060 \\
\hline \multicolumn{7}{|l|}{ Serostatus of partners ${ }^{\mathrm{a}}$} \\
\hline One or more HIV + Partner/s & $400(52)$ & $319(51)$ & $367(52)$ & $335(55)$ & 1.7 & 0.63 \\
\hline One or more HIV- Partner/s & $391(51)$ & 307 (49) & $327(47)$ & $299(49)$ & 2.5 & 0.48 \\
\hline One or more partners of unknown serostatus & $228(30)$ & $177(29)$ & $237(34)$ & $205(34)$ & 6.4 & 0.09 \\
\hline \multicolumn{7}{|l|}{ Type of sexual activity ${ }^{a}$} \\
\hline Any vaginal sex & 257 (77) & $260(82)$ & $317(84)$ & $228(85)$ & 7.4 & 0.070 \\
\hline Any anal insertive sex & $272(35)$ & $214(34)$ & $226(32)$ & $254(41)$ & 15.0 & 0.002 \\
\hline Male sexual partner(s) & $247(54)$ & $192(59)$ & $196(55)$ & $229(63)$ & 8.5 & 0.040 \\
\hline Female sexual partner(s) & $28(16)$ & $27(22)$ & $35(19)$ & $31(30)$ & 8.5 & 0.040 \\
\hline Any anal receptive sex & $315(51)$ & $258(50)$ & $260(47)$ & $299(57)$ & 10.2 & 0.020 \\
\hline \multicolumn{7}{|l|}{ Unprotected Sex in last 6 months } \\
\hline Any unprotected vaginal or anal sex & $309(29)$ & $263(34)$ & $263(27)$ & $322(43)$ & 55.6 & 0.001 \\
\hline \multicolumn{7}{|l|}{ Any unprotected vaginal/anal sex with ${ }^{\mathrm{b}}$} \\
\hline HIV-infected partner & $205(51)$ & $164(51)$ & $162(43)$ & $206(61)$ & 25.0 & 0.001 \\
\hline HIV-uninfected partner & $97(25)$ & $96(31)$ & $88(27)$ & 109 (37) & 12.6 & 0.006 \\
\hline Unknown serostatus partner & $82(36)$ & $74(42)$ & $87(37)$ & $105(51)$ & 13.2 & 0.005 \\
\hline \multicolumn{7}{|l|}{ Injection drug use behavior in last 30 days $^{c}$} \\
\hline Injected any drug in past 30 days $^{\mathrm{c}}$ & $50(5)$ & $30(4)$ & $46(5)$ & $42(6)$ & 2.6 & 0.46 \\
\hline \multicolumn{7}{|l|}{ Times injected in past 30 days $^{\mathrm{d}}$} \\
\hline Mean (SD) & $10.7(14.6)$ & $13.2(18.5)$ & $10.1(16.6)$ & $18.2(29.3)$ & & \\
\hline Median [IQR] & $4.5(3-12)$ & $5(2-20)$ & $4(2-10)$ & $7.5(2-15)$ & & \\
\hline Lent used paraphernalia & $1(2)$ & $3(10)$ & $3(7)$ & $6(14)$ & 5.1 & 0.16 \\
\hline Alcohol use in last 3 months & & & & & 5.2 & 0.81 \\
\hline None & $397(38)$ & $282(37)$ & $382(39)$ & $292(39)$ & & \\
\hline Less than daily & $585(55)$ & $442(58)$ & $534(55)$ & $416(55)$ & & \\
\hline Daily & $51(5)$ & $28(4)$ & $45(5)$ & $32(4)$ & & \\
\hline Unknown/no answer & $22(2)$ & $16(2)$ & $14(1)$ & $18(2)$ & & \\
\hline Stimulant use in last 3 months & & & & & 91.3 & $<0.001$ \\
\hline No & $866(82)$ & $628(82)$ & $755(77)$ & $560(74)$ & & \\
\hline Cocaine only & $121(11)$ & $88(11)$ & $153(16)$ & $77(10)$ & & \\
\hline Speed only & $32(3)$ & $28(4)$ & $36(4)$ & $78(10)$ & & \\
\hline Cocaine and speed & $31(3)$ & $18(2)$ & $26(3)$ & $29(4)$ & & \\
\hline Unknown/no answer & $5(0)$ & $6(1)$ & $5(1)$ & $14(2)$ & & \\
\hline Other drug use in last 3 months & & & & & 18.5 & $<0.005$ \\
\hline No & 799 (76) & $555(72)$ & $699(72)$ & $533(70)$ & & \\
\hline Yes & $251(24)$ & 207 (27) & $271(28)$ & $211(28)$ & & \\
\hline Unknown/No answer & $5(0)$ & $6(1)$ & $5(1)$ & $14(2)$ & & \\
\hline
\end{tabular}

${ }^{a}$ Among those who are sexually active

b Among those with HIV-positive, HIV-uninfected or HIV-unknown status partners respectively

c Among all participants

d Among those who injected drugs in the past 30 days 
anal sex with an unknown status partner. Twenty-one percent of patients reported transmission risk acts, i.e. unprotected anal or vaginal intercourse with an HIVuninfected or unknown status partner in the last 6 months.

\section{Patient Characteristics by Study Group Assignment}

Overall, $30 \%$ of participants were assigned to the standard of care, $22 \%$ to a medical care provider-delivered intervention, $27 \%$ to a prevention specialist-delivered intervention and $21 \%$ to an intervention site using both medical care providers and prevention specialists to deliver intervention messages (multi-provider intervention). We observed demographic and clinic differences in patients assigned to each intervention type (Table 2). Compared to those assigned to standard of care, participants in the medical care provider-delivered interventions were more likely to be women $\left(\chi^{2}=22.1 ; P<0.001\right)$, younger than 40 years of age $\left(\chi^{2}=32.2 ; P<0.001\right)$, African American $\left(\chi^{2}=76.7 ; P<0.001\right)$, to have a paying job $\left(\chi^{2}=9.6\right.$; $P<0.003)$ and to report having a detectable viral load $\left(\chi^{2}=15.9 ; \quad P<0.001\right)$. Participants in the prevention specialist-delivered intervention group were more likely than those in the standard of care to be 40 years of age or older $\left(\chi^{2}=4.1 ; \quad P<0.04\right)$, to have a paying job $\left(\chi^{2}=10.2 ; P<0.006\right)$ and to report having an undetectable viral load $\left(\chi^{2}=18.5 ; P<0.001\right)$. Participants in the multi-provider intervention group were more likely than participants assigned to the standard of care to be younger than 40 years of age $\left(\chi^{2}=24.3 ; P<0.001\right)$ and to report having a detectable viral load $\left(\chi^{2}=17.7 ; P<0.004\right)$. Participants in the multi-provider intervention group were less likely than participants assigned to standard of care to report current use of antiretroviral therapy $\left(\chi^{2}=9.4 ; P<0.001\right)$.

There were also differences in the sexual behavior of patients receiving prevention different intervention providers (Table 3). Compared to patients in the standard of care group, patients in the medical provider-delivered and prevention specialist-delivered intervention groups reported similar sexual transmission risk behavior at baseline. Patients in medical provider-delivered intervention group were more likely to be sexually active $\left(\chi^{2}=15.4\right.$, $P<0.001)$ and to report any unprotected vaginal or anal sex at baseline $\left(\chi^{2}=5.3, P<0.001\right)$, while patients in prevention specialist-delivered intervention group were significantly more likely to report any vaginal sex and sex with an HIV-infected partner $\left(\chi^{2}=5.0, \quad P<0.03\right)$. Patients assigned to receive intervention services from medical care providers and prevention specialists did not differ from those in the standard of care group with respect to unprotected vaginal or anal sex with an HIV-negative or unknown status partner.
In contrast, compared to patients in the standard of care group, patients in the multi-provider intervention group report substantially more sexual transmission risk at baseline. Patients in the multi-provider intervention group were significantly more likely to report being sexually active $\left(\chi^{2}=18.0, P<0.001\right)$, to have two or more sex partners $\left(\chi^{2}=5.1, P<0.02\right)$, to report any vaginal $\left(\chi^{2}=5.4\right.$, $0.02)$ or anal sex $\left(\chi^{2}=5.7,0.02\right)$ with male $\left(\chi^{2}=7.2\right.$, $P<0.008)$ or female $\left(\chi^{2}=8.0, P<0.005\right)$ sex partners, as well as any unprotected vaginal or anal $\operatorname{sex}\left(\chi^{2}=36.1\right.$, $P<0.004)$ and unprotected vaginal or anal sex with HIVinfected $\left(\chi^{2}=8.5, P<0.004\right)$, HIV-uninfected $\left(\chi^{2}=11.0\right.$, $P<0.001)$ and unknown HIV-serostatus $\left(\chi^{2}=10.2\right.$, $P<0.001)$ partners.

\section{Retention and Completion Rates}

At the 12-month follow-up assessment, 58\% of patients were retained in the standard of care group, $76 \%$ of patients were retained in the medical provider intervention group; $62 \%$ were retained in the prevention specialist group and $44 \%$ in the multi-provider group. There were differences in retention by patient characteristics. Older, white, gay patients with more than a high school education but who did not use cocaine or injection drugs were more likely to be retained in the study at 12 -months.

\section{Sexual Transmission Risk Over Time by Intervention Assignment}

Figure 1 illustrates transmission risk across time points for the intervention and comparison arms. We observed a statistically significant effect of prevention-specialist-led interventions at 6 months and medical provider-led interventions at 12 months. Compared to the standard of care group, patients in the medical provider-delivered interventions reported a small effect of the intervention at 6 months (Odds Ratio [OR] $=0.93$; 95\% Confidence Interval $[\mathrm{CI}]=0.60,1.20 ; P<0.79)$ and a much greater effect at 12 months $(\mathrm{OR}=0.55 ; 95 \% \mathrm{CI}=0.32,0.94$; $P<0.03)$. In contrast, compared to patients assigned to the standard of care, patients in the specialist-delivered interventions reported a larger effect of the intervention at 6 months $(\mathrm{OR}=0.58 ; 95 \% \mathrm{CI}=0.35,0.96 ; P<0.04)$, but this effect diminished at 12 months $(\mathrm{OR}=0.67 ; 95 \%$ $\mathrm{CI}=0.39,1.14 ; P<0.14)$. Participants in the multi-provider interventions also reported a small reduction in transmission risk behavior at $6 \quad(\mathrm{OR}=0.89 ; 95 \%$ $\mathrm{CI}=0.53,1.51 ; P<0.68)$ and 12 months $(\mathrm{OR}=0.89$ (95\% $\mathrm{CI}=0.53,1.51 ; P<0.68)$ relative to those in the standard of care group, but these differences were not statistically significant. 


\section{Discussion}

HIV prevention with positives programs delivered by either medical care providers or HIV prevention specialists are effective in reducing sexual risk behavior among patients seen in clinical settings. In this large, multi-site study, messages delivered by HIV prevention specialists influenced behavior change in the shorter term and medical care provider-delivered messages were effective after they had been in place for several months. This study supports others demonstrating the effectiveness of provider-delivered health promotion messages [15].

This study also illustrates the importance of tailoring behavioral interventions to specific clinical settings. Although prevention specialist-led interventions were less likely to influence longer term behavior changes, they did create meaningful change at 6-months and may be a good option for some clinics. The difference in sustained outcomes may be due to factors associated with implementation. In provider sites, patients likely received interventionrelated counseling at each medical visit, with more visits likely to occur and more visits likely to be made closer to the 12-month assessment point. In prevention specialist sites, patients usually received counseling in the days or weeks immediately following the baseline assessment. Our results suggest that behavioral interventions are most effective if they are delivered in "doses"-such as at routine medical care visits-over time, which has been noted in other studies [16].

The sites using a combination of medical care providers and prevention specialists ("multi-provider" sites) did not demonstrate effectiveness in terms of reduced risk among patients receiving intervention services. There is evidence from the qualitative results from this study that these interventions may be difficult to implement because of the attention required to train and sustain provider attention [11]. When a project had to ensure fidelity to an intervention protocol for both medical care providers and prevention specialists within one clinical setting, the effort for project staff was significant and at times, the procedures were too daunting and/or confusing. It may have been that the level of resources available for this intervention study was not great enough to support such complex interventions. It may also be that the complexity of behavioral interventions combined with supporting different types of providers in one setting was just too difficult. In any case, future studies may want to explore issues of quality assurance and efficiency in prevention with positives projects involving more than one type of provider in a clinical setting.

Finally, it is important to note that risk assessment alone seems to influence risk reduction among patients. As has been noted in other studies [16, 17], study participants who were not exposed to any intervention at all still reported reduced risk during ACASI assessments at 6- and 12months. Lightfoot and colleagues have specifically documented the effects of self-monitoring via ACASI on changes in risk behaviors and attitudes [18]. It appears that simply asking patients to report their risk provides an opportunity for reflection that may serve as a cue for selfmotivated change.

Conclusions drawn from this study are limited to some degree by the characteristics of the patients who participated. Most had been infected for 10 years or more and were adherent to both ARVs and to medical care seeking. Patients with these characteristics may have been more likely to also adhere to recommendations from prevention providers. Furthermore, the amount of risk reported by these patients was fairly low and the degree of change possible was therefore limited. Nevertheless, our study yielded significant results among patients receiving medical care provider- and prevention specialist-delivered interventions. There would be a greater magnitude of change possible with riskier patients and thus, our conclusions are likely conservative.

Conclusions are also limited by lack of standardization of intervention content; each site chose and tailored its own intervention. However, we are able to assure some level of commonality across the types we included in this analysis because sites were required to create and implement quality assurance plans to ensure fidelity to the intervention protocols. Study staff used a variety of techniques including provider interview, observation and quizzes to ensure that providers of all kinds adhered to the study protocols. In addition, because this study provides data from "real world" settings and implementation, the chances of replication of proven-effective interventions are enhanced.

Another limitation of our study is that the two sites using medical care provider-delivered messages alone also employed the use of computer-assisted risk assessment. There is some evidence from the qualitative study [19] that medical care providers remain uncomfortable with in-depth discussions of patients' sexual behavior. For this reason, it is possible that the same degree of change would not have been found if providers and not computers had conducted the risk assessment. On the other hand, it does appear that interventions using computer-aided risk assessment in combination with medical-care provider delivered messages are an effective strategy for prevention with positives programs. This finding supports other recently emerging studies, such as those published by Gilbert and colleagues [20] who note the value of a "Video Doctor." In the Video Doctor intervention, the computer performed both the risk assessment and the counseling, with durable risk reduction effects. Nevertheless, medical care providers in this study welcomed the opportunity to discuss risk reduction with 
their patients, an unintended consequence which cannot be underestimated.

This study contributes to the growing evidence regarding the effectiveness of prevention interventions for HIVinfected people in clinical settings. Our results support increased calls for the integration of prevention into care settings and for more research to understand how intervention "fit" contributes to program success. This is the first published cross-site outcomes study from the SPNS Prevention with Positives Initiative. Forthcoming papers describing the findings of the qualitative study and the cost effectiveness of interventions will provide additional evidence for selection and implementation of prevention models.

Acknowledgements This publication was supported by the Health Resources and Services Administration (HRSA) Special Projects of National Significance (SPNS) Program (grant number 5 H97 HA00261). The publication's contents are solely the responsibility of the authors and do not necessarily represent the official view of HRSA or the SPNS program. We would like to express our appreciation to Jay Newberry and Stuart Gaffney for their support over the course of the project and during the drafting of this paper.

Open Access This article is distributed under the terms of the Creative Commons Attribution Noncommercial License which permits any noncommercial use, distribution, and reproduction in any medium, provided the original author(s) and source are credited.

\section{References}

1. Gardner LI, Marks G, O'Daniels CM, et al. Implementation and evaluation of a clinic-based behavioral intervention: positive steps for patients with HIV. AIDS Patient Care STDS. 2008;22(8):627-35.

2. Richardson JL, Milam J, McCutchan A, et al. Effect of brief safer-sex counseling by medical providers to HIV-1 seropositive patients: a multi-clinic assessment. AIDS. 2004;18(8):1179-86.

3. Fisher JD, Fisher WA, Cornman DH, Amico RK, Bryan A, Friedland GH. Clinician-delivered intervention during routine clinical care reduces unprotected sexual behavior among HIV-infected patients. J Acquir Immune Defic Syndr. 2006;41(1):44-52.

4. Lightfoot M, Rotheram-Borus MJ, Tevendale H. An HIV-preventive intervention for youth living with HIV. Behav Modif. 2007;31(3):345-63.
5. Kalichman S, Rompa D, Cage M, et al. Effectiveness of an intervention to reduce HIV transmission risks in HIV-positive people. Am J Prev Med. 2001;21(2):84-92.

6. McKirnan DJ, Swanson F, Tolu-Shams M, Ramey B, Flynn J. Howard Brown Health Center. Effectiveness of a general coping approach to HIV adherence. Paper presented at: Society of Behavioral Medicine Conference; Seattle, Washington, 2001.

7. Malitz FE, Eldred L. Evolution of the special projects of national significance prevention with HIV-infected persons seen in primary care settings initiative. AIDS Behav. 2007;11(5 Suppl):S1-5.

8. Prochaska JO, DiClemente CC. Stages of change in the modification of problem behaviors. Prog Behav Modif. 1992;28:183218.

9. Miller WR, Rollnick S. Motivational interviewing: preparing people to change. New York, NY: Guilford Press; 2002.

10. Alan Marlatt G. Harm reduction: pragmatic strategies for managing high-risk behaviors. New York, NY: Guilford Press; 2002.

11. Koester KA, Maiorana A, Vernon K, Myers JJ, Dawson Rose C, Morin SF. Implementation of HIV prevention interventions with people living with HIV/AIDS in clinical settings: challenges and lessons learned. AIDS Behav. 2007;11(5):S17-29.

12. Robins JM, Hernan MA, Brumback B. Marginal structural models and causal inference in epidemiology. Epidemiology. 2000;11(5):550-60.

13. Hernan MA, Brumback B, Robins JM. Marginal structural models to estimate the causal effect of zidovudine on the survival of HIV-positive men. Epidemiology. 2000;11(5):561-70.

14. Breslow NE, Day NE. Statistical methods in cancer research, vol II: the design and analysis of cohort studies. New York, NY: Oxford University Press; 1987.

15. Whitlock EP, Orleans CT, Pender N, Allan J. Evaluating primary care behavioral counseling interventions: an evidence-based approach. Am J Prev Med. 2002;22:267-84.

16. Healthy Living Project Team. Effects of a behavioral intervention to reduce risk of transmission among people living with HIV: the healthy living project randomized controlled study. J Acquir Immune Defic Syndr. 2007;44(2):213-21.

17. Kamb ML, Fishbein M, Douglas JM Jr, et al. Efficacy of riskreduction counseling to prevent human immunodeficiency virus and sexually transmitted diseases: a randomized controlled trial. Project RESPECT Study Group. JAMA. 1998;280:1161-7.

18. Lightfoot M, Rotheram-Borus MJ, Comulada S, Gundersen G, Reddy V. Self-monitoring of behaviour as a risk reduction strategy for persons living with HIV. AIDS Care. 2007;19(6):757-63.

19. Koester KA, Maiorana A, Morin SF, Dawson Rose C, Shade S, Myers JJ. Patients' perspectives on HIV prevention with positives interventions in clinical settings: "I feel like a huge weight has been lifted off of me". Soc Sci Med. (under review).

20. Gilbert P, Ciccarone D, Gansky SA, et al. Interactive "Video Doctor" counseling reduces drug and sexual risk behaviors among HIV-positive patients in diverse outpatient settings. PLoS ONE. 2008;3(4):e1988. 